\title{
Evidence of Nephropathy and Peripheral Neuropathy in US Adults With Undiagnosed Diabetes
}

\author{
Richelle J. Koopman, MD, MS ${ }^{1}$ \\ Arch G. Mainous III, PbD ${ }^{1}$ \\ Heather A. Liszka, MD, MS \\ Jobn A. Colwell, MD, $P b D^{2}$ \\ Elizabeth H. Slate, $\mathrm{PbD}^{3}$ \\ Mark A. Carnemolla, BS \\ Charles J. Everett, $P b D^{1}$ \\ 'Department of Family Medicine, \\ Medical University of South Carolina, \\ Charleston, SC \\ ${ }^{2}$ Department of Medicine, Division of \\ Endocrinology, Diabetes, and Medical \\ Genetics, Medical University of South \\ Carolina, Charleston, SC
}

${ }^{3}$ Department of Biostatistics, Bioinformatics, and Epidemiology, Medical University of South Carolina, Charleston, SC

\begin{abstract}
PURPOSE Nearly one third of diabetes cases in the United States is undiagnosed, with mounting evidence that complications accrue even before clinical diagnosis. We wanted to determine whether persons with undiagnosed diabetes have signs of nephropathy and peripheral neuropathy

METHODS We examined the prevalence of positive screening tests for nephropathy and peripheral neuropathy in adults aged $\geq 40$ years with undiagnosed diabetes using secondary analysis of survey and examination data from the population-based United States National Health and Nutrition Examination Survey 1999-2002. We defined a positive screening test for nephropathy as a spot urine albumin-creatinine ratio $>30.0 \mathrm{mg} / \mathrm{g}$, representing at least microalbuminuria. We defined $\geq 1$ insensate area on Semmes-Weinstein monofilament testing as a positive finding for neuropathy. Undiagnosed diabetes was defined as a combination of no history of diagnosed diabetes and a measured fasting glucose $\geq 126 \mathrm{mg} / \mathrm{dL}$. We used SUDAAN for $\chi^{2}$ and regression analyses.
\end{abstract}

RESULTS The prevalence of a positive test when screening for nephropathy among those with undiagnosed diabetes was $26.5 \%$ compared with $7.1 \%$ in those with no diabetes $\left(\chi^{2}, P<.01\right)$. After adjusting for age and diagnosed or undiagnosed hypertension, the association of undiagnosed diabetes with nephropathy persisted (odds ratio $=2.35 ; 95 \%$ confidence interval, $1.38-4.01$ ). For peripheral neuropathy, $21.5 \%$ with undiagnosed diabetes had positive screening tests compared with $10.1 \%$ with no diabetes $\left(\chi^{2}, P<.01\right)$; however, this effect was not significant after adjustment for age. There was no significant difference in positive screening tests for nephropathy or neuropathy when comparing those with undiagnosed and diagnosed diabetes.

CONCLUSIONS A significant proportion of adults with undiagnosed diabetes have signs of nephropathy and peripheral neuropathy. These findings may influence policies about early screening for diabetes.

Ann Fam Med 2006:4;427-432. DOI: 10.1370/afm.577.

\section{INTRODUCTION}

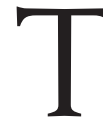
The prevalence of undiagnosed diabetes for US adults has been estimated at $2.4 \%$, representing 4.9 million adults and nearly $30 \%$ of all cases of diabetes in the United States in 1999-2000. ${ }^{1}$ Type 2 diabetes is especially likely to be undiagnosed for years because of its insidious clinical prodrome; projections based on extrapolation of time to development of complications of diabetes estimate that the average time from the onset of disease to clinical diagnosis of diabetes in the US population is as much as 12 years. ${ }^{2,3}$ Twenty percent of persons with undiagnosed diabetes already have developed retinopathy. ${ }^{2}$ The prevalence of other microvascular complications among US adults with undiagnosed diabetes is unknown, although microvascular complications, including retinopathy and signs of 
nephropathy and neuropathy, were found to be highly prevalent at the time of diagnosis of diabetes in United Kingdom and Dutch populations. ${ }^{4,5}$

Peripheral sensory neuropathy is an important complication of diabetes and a major contributor to diabetic foot ulcers. ${ }^{6}$ In the United States in 1999-2000, the prevalence of peripheral neuropathy, as measured by at least 1 insensate area on monofilament testing, has been found to be $28.5 \%$ in those aged 40 years and older with diagnosed diabetes. ${ }^{7}$

Diabetes is the leading cause of end-stage renal disease in the United States. ${ }^{8,9}$ The prevalence of one of the earliest signs of nephropathy, microalbuminuria, in the United States among persons with diagnosed diabetes has been estimated at $28.1 \%$, with $6.1 \%$ exhibiting macroalbuminuria. ${ }^{10}$

Using a population-based sample, we describe the population prevalence of positive screening tests for 2 microvascular complications of diabetes, nephropathy and peripheral neuropathy, among adults aged 40 years and older with undiagnosed diabetes in the United States in 1999-2002. This knowledge will broaden our understanding of the early complications of diabetes that are present even before clinical diagnosis.

\section{METHODS}

\section{Data Source}

The most recent National Health and Nutrition Examination Survey (NHANES), a product of the National Center for Health Statistics, spans the years 1999-2002 and includes a household interview, dietary interview, physician examination, and laboratory and diagnostic testing at a Mobile Examination Center. The NHANES 1999-2002 uses a stratified, multistage probability sample, allowing estimation of disease prevalence in the civilian, noninstitutionalized US population. We sampled adults 40 years of age and older for this analysis because this population in the NHANES had both monofilament and urine testing. We also used the subsample of participants who had fasting plasma glucose measurements, resulting in an unweighted sample size of 2,571. For the analysis of peripheral neuropathy, our sample size is slightly smaller because not all adults had monofilament testing. Additionally, because participants who reported a history of stroke $(n=103)$ were excluded from the neuropathy sample, as stroke might confound the assessment of peripheral sensation, the resulting final sample size was 2,337. The Institutional Review Board of the Medical University of South Carolina approved this research.

\section{Nephropathy: Urinary Albumin-Creatinine Ratio} Both urine albumin and urine creatinine were measured in NHANES 1999-2002 adult participants using a Jaffe rate reaction for creatinine analysis and solidphase fluorescent immunoassay for albumin analysis. ${ }^{11}$ The spot urine albumin-creatinine ratio is recommended by the American Diabetes Association to screen for diabetic nephropathy and is less susceptible to false negatives and false positives than is spot urine albumin testing without concomitant urine creatinine measurement. ${ }^{12,13}$ A spot urine albumin-creatinine ratio $\geq 30 \mathrm{mg} / \mathrm{g}$ is defined as abnormal, representing at least microalbuminuria. ${ }^{13,14}$

Peripheral Neuropathy: Insensate Area on 5.07 Semmes-Weinstein Monofilament Testing Loss of protective sensation was ascertained by a standard monofilament test for NHANES participants aged 40 years and older. ${ }^{15}$ Slight pressure with a 5.07 Semmes-Weinstein monofilament was applied 3 times to each of 3 areas on each foot (hallux and first and fifth metatarsal heads). The testing used a 2 -alternative forced-choice algorithm. ${ }^{16}$ Insensitivity at any site on more than 1 occasion constitutes a positive screening test for peripheral neuropathy. Further details of the NHANES testing procedure are available. ${ }^{17}$ Positive 5.07 Semmes-Weinstein monofilament testing in persons with diabetes has been shown to be predictive of future diabetic foot ulceration, and it has been used previously to estimate the prevalence of peripheral neuropathy in populations. $7,18,19$

\section{Diagnosed Diabetes, Undiagnosed Diabetes, No Diabetes}

The NHANES assessed participants for diagnosed diabetes using the question, "Other than during pregnancy, have you ever been told by a doctor or health professional that you have diabetes or sugar diabetes?" We defined participants as having diagnosed diabetes if they answered "yes" to this question. We defined participants as having undiagnosed diabetes if they answered "no" to this question and had a fasting plasma glucose of $\geq 126 \mathrm{mg} / \mathrm{dL}$ ( $7.0 \mathrm{mmol} / \mathrm{L})$. We defined participants as having no diabetes if they did not have laboratory evidence consistent with diabetes and denied a history of diagnosed diabetes. This method of classifying NHANES participants as having diagnosed, undiagnosed, and no diabetes has been used previously. ${ }^{1}$

As a check on the validity of the classification of undiagnosed diabetes, we examined the medication lists of the NHANES participants whom we defined as having no diabetes or undiagnosed diabetes for evidence of use of medicines classified according to the US Food and Drug Administration's National Drug Code Directory (NDC). ${ }^{20}$ Participants were reclassified as having diagnosed diabetes if they reported being on a medicine in NDC drug class code 1036, "blood glucose regula- 
tors," which accounted for $0.5 \%$ of the total sample of those initially classified as having undiagnosed diabetes.

We investigated demographic characteristics of the population with diagnosed, undiagnosed, and no diabetes, including age, sex, and race/ethnicity. Race/ethnicity is self-defined by NHANES participants according to the following classifications: non-Hispanic white, non-Hispanic black, Hispanic, and other. We also investigated the health care access factors of lack of health insurance, absence of a regular place of care, and lack of any health care utilization during the past year, as well as the socioeconomic factors of low income, defined as less than $\$ 20,000$ for the household for the year, high school education (less than, more than, or equivalent), and marital status. Health insurance was assessed only for those aged less than 65 years, as health care coverage in the United States for those aged 65 years and up is nearly universal as a result of the Medicare program. We examined the participants' body mass index (BMI) and self-rated health status, which had the possible values of excellent, very good, good, fair, or poor.

Additionally we examined the prevalence of diagnosed and undiagnosed hypertension in the sample, because there is a potential confounding effect of hypertension on the relationship between diabetes and nephropathy/microalbuminuria. Participants were classified as having diagnosed hypertension if they answered "yes" to the NHANES survey question, "Have you ever been told by a doctor or other health professional that you had hypertension, also called high blood pressure?" In a manner similar to defining participants with undiagnosed diabetes, we defined individuals as having undiagnosed hypertension if they answered "no" to this question about diagnosed hypertension and if they had either a measured systolic blood pressure $\geq 140 \mathrm{~mm} \mathrm{Hg}$ or a measured diastolic blood pressure $\geq 90 \mathrm{~mm} \mathrm{Hg}$. Blood pressure in the NHANES survey was the average of 3 to 4 blood pressures measured with a mercury sphygmomanometer according to the American Heart Association recommended procedures. ${ }^{21}$ Finally, we examined the medication lists of the NHANES participants whom we defined as having no evidence of hypertension or undiagnosed hypertension for evidence of medicines classified according to the US Food and Drug Administration's NDC. ${ }^{20}$ Participants were reclassified as having diagnosed hypertension if they reported being on a medicine in NDC drug class codes 0506 "antihypertensives," 0507 "diuretics," 0510 "calcium channel blockers," 0512 "beta blockers," 0513 "alpha agonist/ alpha blockers," and 0514 "ace inhibitors." NHANES interviewers also ascertained the main reason for the use of the medication, further validating the classification of each medication as an antihypertensive.

\section{Analysis}

We applied appropriate sample weights to the unweighted samples to calculate population estimates of those with a positive screening test according to their diabetes diagnosis status. We used sample weights for the subpopulation with fasting glucose measurements and the combined sample weights for the 19992000 and 2001-2002 data releases, as recommended for NHANES analysis. ${ }^{22}$ We compared rates of a positive screening test for nephropathy and for peripheral neuropathy in those with no evidence of diabetes, undiagnosed diabetes, and diagnosed diabetes using a $\chi^{2}$ test with SUDAAN statistical software for this clustered and weighted probability sample. ${ }^{23}$

Because hypertension and undiagnosed hypertension are common in persons with diagnosed and undiagnosed diabetes in this sample, it is possible that the presence of hypertension might confound the effect of diagnosed and undiagnosed diabetes on microalbuminuria. ${ }^{5}$ Additionally, age is related to both the outcomes of nephropathy and neuropathy. ${ }^{7}$ We therefore used logistic regression to examine the effect of undiagnosed diabetes on the presence of microalbuminuria after adjustment for age and hypertension, comprising both diagnosed and undiagnosed hypertension. We also used logistic regression to examine the effect of undiagnosed diabetes on a positive screening test for peripheral neuropathy after adjustment for age.

\section{RESULTS}

The sociodemographic and health care access characteristics of those aged 40 years and older with undiagnosed diabetes in the United States in 1999-2002, as opposed to those with diagnosed diabetes and no evidence of diabetes, are displayed in Table 1. There were 399 people in the unweighted sample with an elevated urine albumin-creatinine ratio and 332 people in the unweighted sample with at least 1 area of insensitivity on either foot and no history of stroke.

The prevalence of positive screening tests for nephropathy and peripheral neuropathy among US adults aged 40 years and older with no evidence of diabetes, undiagnosed diabetes, and diagnosed diabetes is represented in Table 2 . When compared only with those with no evidence diabetes, those who had undiagnosed diabetes had significantly more nephropathy, $P<.01\left(\chi^{2}\right)$; when compared with those who had diagnosed diabetes, there was no significant difference, $P=.63\left(\chi^{2}\right)$. Similarly, a significantly greater proportion of persons with undiagnosed diabetes had a positive screening test for peripheral neuropathy than did those with no evidence of diabetes, $P=.04\left(\chi^{2}\right)$, whereas there was no significant difference between the groups 
with undiagnosed and previously diagnosed diabetes, $P=.72\left(\chi^{2}\right)$. In an analysis of positive screening tests for peripheral neuropathy, including those with stroke, a similar pattern was found, with significantly more screening tests positive for peripheral neuropathy in those with undiagnosed diabetes than in those with no evidence of diabetes, $P=.02\left(\chi^{2}\right)$, whereas there was no significant difference in positive screening tests for peripheral neuropathy between the groups with undiagnosed and previously diagnosed diabetes.

In a logistic regression model examining the relative contributions of diabetes, undiagnosed diabetes,

\begin{tabular}{|c|c|c|c|}
\hline Factors & $\begin{array}{l}\text { Diagnosed } \\
\text { Diabetes }\end{array}$ & $\begin{array}{l}\text { Undiagnosed } \\
\text { Diabetes }\end{array}$ & $\begin{array}{c}\text { No } \\
\text { Evidence } \\
\text { of Diabetes }\end{array}$ \\
\hline Unweighted sample size, $n$ & 271 & 132 & 2,168 \\
\hline $\begin{array}{l}\text { Weighted population size } \\
\text { (millions), } \mathrm{n}\end{array}$ & 11.4 & 4.8 & 101.2 \\
\hline Weighted prevalence, \% & 9.7 & 4.1 & 86.3 \\
\hline \multicolumn{4}{|l|}{ Age, years } \\
\hline $40-64$ & 62.4 & 64.1 & 76.0 \\
\hline$\geq 65$ & 37.7 & 35.9 & 24.0 \\
\hline Sex, male, \% & 54.3 & 63.2 & 45.5 \\
\hline \multicolumn{4}{|l|}{ Race/ethnicity, \% } \\
\hline Non-Hispanic white & 65.2 & 77.2 & 79.0 \\
\hline Non-Hispanic black & 11.5 & 11.6 & 9.0 \\
\hline Hispanic & 17.4 & 8.0 & 8.9 \\
\hline Other & 5.9 & 3.2 & 3.1 \\
\hline No health care utilization, \% & 3.8 & 15.3 & 12.5 \\
\hline No usual place of care, \% & 3.9 & 12.1 & 11.0 \\
\hline No health insurance, \%* & 8.1 & 22.4 & 12.7 \\
\hline Income $<\$ 20,000 / y, \%$ & 34.2 & 35.8 & 21.9 \\
\hline \multicolumn{4}{|l|}{ Education, \% } \\
\hline$<$ High school & 31.5 & 36.7 & 21.2 \\
\hline High school & 21.8 & 22.8 & 24.4 \\
\hline > High school & 46.8 & 40.5 & 54.4 \\
\hline Body mass index $\geq 30 \mathrm{~kg} / \mathrm{m}^{2}, \%$ & 46.5 & 55.4 & 29.1 \\
\hline Not married, \% & 34.8 & 45.5 & 31.7 \\
\hline \multicolumn{4}{|l|}{ Self-rated health status, $\%$} \\
\hline Fair/poor & 37.8 & 24.3 & 18.9 \\
\hline \multicolumn{4}{|l|}{ Hypertension } \\
\hline Diagnosed & 65.1 & 60.9 & 39.4 \\
\hline Undiagnosed & 8.6 & 15.9 & 10.2 \\
\hline
\end{tabular}

Table 2. Prevalence of Positive Screening Tests for Nephropathy and Peripheral Neuropathy in US Adults Aged $\geq 40$ Years With Diagnosed Diabetes, Undiagnosed Diabetes, and No Diabetes

\begin{tabular}{lccc}
\hline Screening Test & $\begin{array}{c}\text { No Diabetes } \\
\% \text { (SE) }\end{array}$ & $\begin{array}{c}\text { Undiagnosed } \\
\text { Diabetes } \\
\% \text { (SE) }\end{array}$ & $\begin{array}{c}\text { Diagnosed } \\
\text { Diabetes } \\
\% \text { (SE) }\end{array}$ \\
\hline Nephropathy & $9.6(0.6)^{*}$ & $24.9(4.4)$ & $28.0(3.7)$ \\
Peripheral neuropathy ${ }^{\dagger}$ & $10.1(0.9)^{*}$ & $21.5(5.1)$ & $19.2(3.4)$ \\
\hline * Difference vs undiagnosed diabetes group significant at $P<.05$. & \\
† Estimates for peripheral neuropathy exclude participants with a history of stroke.
\end{tabular}
age, and any hypertension to the presence of microalbuminuria, the effect of both undiagnosed diabetes (odds ratio $[\mathrm{OR}]=2.35 ; 95 \%$ confidence interval [CI $], 1.38-4.01)$ and diagnosed diabetes $(\mathrm{OR}=3.01$; 95\% CI, 1.99-4.57) persisted even after adjustment for age and diagnosed or undiagnosed hypertension. In a logistic regression model examining the contributions of diabetes and undiagnosed diabetes to the presence of a positive screening test for peripheral neuropathy, with adjustment for age, diagnosed diabetes was a significant predictor $(\mathrm{OR}=1.72 ; 95 \% \mathrm{CI}, 1.01-2.91)$; however, undiagnosed diabetes was not $(\mathrm{OR}=1.90 ; 95 \% \mathrm{CI}, 0.91-3.96)$.

\section{DISCUSSION}

This study is the first examination of the prevalence of positive screening tests for nephropathy and peripheral neuropathy among those who had undiagnosed diabetes in a US population-based sample.

That $24.9 \%$ of adults 40 years and older with undiagnosed diabetes had signs of nephropathy and $21.5 \%$ had signs of peripheral neuropathy adds to the accumulating literature which documents undiagnosed diabetes is not a benign condition but represents a serious public health concern. Rates of positive screening tests for these 2 diabetes complications in those with undiagnosed diabetes were both significantly higher than in the nondiabetic population, but these rates were not significantly different from those with diagnosed diabetes. Additionally, the effect of undiagnosed diabetes on the presence of microalbuminuria persisted after adjustment for age and diagnosed or undiagnosed hypertension.

These findings, along with estimates of the prevalence of complications at the time of diagnosis, suggest the time before diagnosis of diabetes is neither 
benign nor quiescent. ${ }^{4,5,24}$ The time from disease onset to clinical diagnosis has been estimated to be at least 9 to 12 years. ${ }^{2,3}$ Furthermore, onset of type 2 diabetes is often insidious, with a prodrome of subclinical disease that may last more than 10 years and involves insulin resistance and other metabolic abnormalities. ${ }^{25-27}$ Insulin resistance in the absence of diabetes has been shown to be a risk factor for cardiovascular disease, prolonging the period of potential risk for complications. ${ }^{28,29}$ In fact, increased prevalence of microalbuminuria and sensory neuropathy is found in those with impaired glucose tolerance. ${ }^{30,31}$

The population estimates for a positive screening test for peripheral neuropathy among persons with diagnosed diabetes in this study are lower than in past published inquiries. ${ }^{7}$ Several reasons account for this difference. First, we excluded participants with stroke. Second, we reclassified persons with no evidence of diabetes or with undiagnosed diabetes who were using diabetes medications. Third, we were using a larger data set that includes not only the 1999-2000 NHANES survey but also the more recent data from the 2001-2002 NHANES survey; the inclusion of these additional data may in itself lead to slight differences in prevalence estimates. Lastly, our investigation used fasting plasma glucose values to define and contrast those with undiagnosed diabetes, which led us to use a morning fasting subsample of NHANES participants and the associated morning subsample weights to reach our population estimates. Similarly, the population estimates of microalbuminuria differ from previous studies for many of the same reasons, including the limitation of our sample to adults aged 40 years and older. ${ }^{10}$

One strength of this study is its ability to produce population estimates for undiagnosed diabetes and its coexisting complications for the US population of adults aged 40 years and older. Only through using the NHANES data set, which simultaneously assesses a history of diabetes with laboratory evidence for diabetes, can we arrive at estimates of undiagnosed diabetes. In this way, we can, in effect, know what is unknown. We were also able to examine some of the sociodemographic and health care access factors associated with undiagnosed diabetes.

A few limitations must be acknowledged. Because our study is a cross-sectional examination, inferences about sociodemographic health care access characteristics among those with undiagnosed diabetes is limited in that cause and effect can not be determined. For example, those with undiagnosed diabetes were less likely to have health insurance than those with previously diagnosed diabetes. Even so, we cannot say whether the lack of insurance resulted in the lack of diagnosis of diabetes or whether, conversely, those with diagnosed diabetes were more likely to subsequently obtain health insurance.

Semmes-Weinstein monofilament testing is a screening test for peripheral neuropathy and does not constitute a definitive diagnosis of neuropathy, although it certainly has documented clinical utility. ${ }^{32}$ Additionally, although it may not be as accurate as nerve conduction studies, monofilament testing has utility for estimates of disease in large epidemiologic samples such as the NHANES, it has been used similarly in previous studies, and it is recommended by the ADA for assessment of the foot in all patients with diabetes who are not already known to have neuropathy. ${ }^{7,12}$ We excluded persons with stroke, but there may be other causes of decreased peripheral sensation that we were not able to address which may affect the prevalence of a positive screening test, particularly among those with no evidence of diabetes. We did not investigate metabolic causes of peripheral neuropathy other than diabetes because of the limitations of using existing data.

Mass population screening for diabetes is not recommended at this time ${ }^{33-35}$ despite evidence that early treatment can prevent complications. ${ }^{36}$ Although the results presented here do not lead us to advocate mass screening, they do sensitize us to the point that the current approach of detecting diabetes once clinical signs and symptoms are apparent may be too late to prevent complications and may be a strategy in need of review. The evidence presented in this study is consistent with similar evidence for retinopathy, ${ }^{2}$ which suggests that those with undetected diabetes are already beginning to accrue complications of diabetes. This new knowledge about the prevalence of positive screening tests for nephropathy and peripheral neuropathy in those with undiagnosed diabetes may have implications for policy and research initiatives regarding screening and detection of diabetes, as well as efforts to prevent diabetes. We have effective, inexpensive, and minimally invasive tests to detect both diabetes and prediabetic states. More aggressive use of screening based on risk stratification for diabetes is in order and should be appropriately reimbursed. Research that focuses on risk stratification may lead to more cost-effective screening strategies. Additional evidence of early complications and investigation into the effects of early detection and treatment of diabetes may further inform decisions about policies to screen based on risk.

To read or post commentaries in response to this article, see it online at http://www.annfammed.org/cgi/content/full/4/5/427.

Key words: Diabetes mellitus/diagnosis; peripheral neuropathies; albuminuria; diabetic nephropathy; diagnosis; NHANES

Submitted December 7, 2005; submitted, revised, February 7, 2006; accepted February 17, 2006. 
Funding support: This study was funded through grant 1 R21 DK067130-01A1 from the National Institute for Diabetes $\&$ Digestive $\&$ Kidney Diseases and through grants 1 D14 HP 00161 and 2 D54 HP00023 from the Health Resources and Services Administration.

\section{References}

1. Prevalence of diabetes and impaired fasting glucose in adults--United States, 1999-2000. MMWR Morb Mortal Wkly Rep. 2003;52:833-837.

2. Harris MI, Klein R, Welborn TA, Knuiman MW. Onset of NIDDM occurs at least 4-7 yr before clinical diagnosis. Diabetes Care. 1992;15:815-819.

3. Jarrett RJ. Duration of non-insulin-dependent diabetes and development of retinopathy: analysis of possible risk factors. Diabet Med. 1986;3:261-263.

4. UK Prospective Diabetes Study 6. Complications in newly diagnosed type 2 diabetic patients and their association with different clinical and biochemical risk factors. Diabetes Res. 1990;13:1-11.

5. Spijkerman AM, Dekker JM, Nijpels G, et al. Microvascular complications at time of diagnosis of type 2 diabetes are similar among diabetic patients detected by targeted screening and patients newly diagnosed in general practice: the hoorn screening study. Diabetes Care. 2003;26:2604-2608.

6. Jeffcoate WJ, Harding KG. Diabetic foot ulcers. Lancet. 2003;361: 1545-1551.

7. Gregg EW, Sorlie P, Paulose-Ram R, et al. Prevalence of lowerextremity disease in the US adult population $\geq 40$ years of age with and without diabetes: 1999-2000 national health and nutrition examination survey. Diabetes Care. 2004;27:1591-1597.

8. US Renal Data System, USRDS 2005 Annual Data Report: Atlas of EndStage Renal Disease in the United States. Bethesda, Md: National Institutes of Health, National Institute of Diabetes and Digestive and Kidney Diseases; 2006.

9. Ritz E, Orth SR. Nephropathy in patients with type 2 diabetes mellitus. N Engl J Med. 1999;341:1127-1133.

10. Garg AX, Kiberd BA, Clark WF, Haynes RB, Clase CM. Albuminuria and renal insufficiency prevalence guides population screening: results from the NHANES III. Kidney Int. 2002;61:2165-2175.

11. Centers for Disease Control and Prevention. National Health and Nutrition Examination Survey. 2001-2002 Data Release, Documentation for Laboratory Results, Laboratory 16 - Urinary Creatinine and Albumin. May 2004. Available at: http://www.cdc.gov/nchs/data/ nhanes/nhanes_01_02/116_b_doc.pdf. Accessed: 28 November 2005

12. Standards of medical care in diabetes. Diabetes Care. 2005 28(Suppl 1):S4-S36

13. Eknoyan G, Hostetter T, Bakris GL, et al. Proteinuria and other markers of chronic kidney disease: a position statement of the national kidney foundation (NKF) and the national institute of diabetes and digestive and kidney diseases (NIDDK). Am J Kidney Dis. 2003:42:617-622.

14. Keane W/F, Eknoyan G. Proteinuria, albuminuria, risk, assessment, detection, elimination (PARADE): a position paper of the National Kidney Foundation. Am J Kidney Dis. 1999;33:1004-1010.

15. Mayfield JA, Reiber GE, Sanders LJ, Janisse D, Pogach LM. Preventive foot care in diabetes. Diabetes Care. 2004;27(Suppl 1):S63-64.

16. Sekuler R, Nash D, Armstrong R. Sensitive, objective procedure for evaluating response to light touch. Neurology. 1973;23:1282-1291.

17. Centers for Disease Control and Prevention. National Health and Nutrition Examination Survey. Lower extremity disease procedures manual. January 2001. Available at: http://www.cdc.gov/nchs/data/ nhanes/le.pdf. Accessed: 28 November 2005.
18. Pham H, Armstrong DG, Harvey C, et al. Screening techniques to identify people at high risk for diabetic foot ulceration: a prospective multicenter trial. Diabetes Care. 2000;23:606-611.

19. Boyko EJ, Ahroni JH, Stensel V, et al. A prospective study of risk factors for diabetic foot ulcer. The Seattle Diabetic Foot Study. Diabetes Care. 1999;22:1036-1042.

20. US Department of Health and Human Services, Food and Drug Administration, Center for Drug Evaluation and Research. National Drug Code Directory. Available at: http://www.fda.gov/cder/ndc/ database/default.htm. Accessed: 28 November 2005.

20. Perloff D, Grim C, Flack J, et al. Human blood pressure determination by sphygmomanometry. Circulation. 1993;88:2460-2470.

22. NHANES Analytic Guidelines. June, 2004. Available at: http://www. cdc.gov/nchs/data/nhanes/nhanes_general_guidelines_june_04.pdf. Accessed: 28 November 2005.

23. SUDAAN. Version 9.0.1 for SAS Version 9. Research Triangle Park, NC: Research Triangle Institute; 2005.

24. Taubert G, Winkelmann BR, Schleiffer T, et al. Prevalence, predictors, and consequences of unrecognized diabetes mellitus in 3266 patients scheduled for coronary angiography. Am Heart J. 2003:145:285-291.

25. Lillioja S, Mott DM, Spraul M, et al. Insulin resistance and insulin secretory dysfunction as precursors of non-insulin-dependent diabetes mellitus. Prospective studies of Pima Indians. N Engl J Med. 1993;329:1988-1992

26. Martin BC, Warram JH, Krolewski AS, et al. Role of glucose and insulin resistance in development of type 2 diabetes mellitus: results of a 25-year follow-up study. Lancet. 1992;340:925-929.

27. Facchini FS, Hua N, Abbasi F, Reaven GM. Insulin resistance as a predictor of age-related diseases. J Clin Endocrinol Metab. 2001;86: 3574-3578.

28. Haffner SM, Stern MP, Hazuda HP, Mitchell BD, Patterson JK. Cardiovascular risk factors in confirmed prediabetic individuals. Does the clock for coronary heart disease start ticking before the onset of clinical diabetes? JAMA. 1990;263:2893-2898.

29. Haffner SM, Mykkanen L, Festa A, Burke JP, Stern MP. Insulin-resistant prediabetic subjects have more atherogenic risk factors than insulin-sensitive prediabetic subjects: implications for preventing coronary heart disease during the prediabetic state. Circulation. 2000;101:975-980.

30. Kim YI, Kim CH, Choi CS, et al. Microalbuminuria is associated with the insulin resistance syndrome independent of hypertension and type 2 diabetes in the Korean population. Diabetes Res Clin Pract. 2001:52:145-152.

31. Franklin GM, Kahn LB, Baxter J, Marshall JA, Hamman RF. Sensory neuropathy in non-insulin-dependent diabetes mellitus. The San Luis Valley Diabetes Study. Am J Epidemiol. 1990;131:633-643.

32. Mayfield JA, Sugarman JR. The use of the Semmes-Weinstein monofilament and other threshold tests for preventing foot ulceration and amputation in persons with diabetes. J Fam Pract. 2000;49:517-29.

33. Screening for type 2 diabetes. Diabetes Care. 2004;27(Suppl 1): S11-14.

34. Screening for type 2 diabetes mellitus in adults: recommendations and rationale. Ann Intern Med. 2003;138:212-214.

35. Harris R, Donahue K, Rathore SS, et al. Screening adults for type 2 diabetes: a review of the evidence for the U.S. Preventive Services Task Force. Ann Intern Med. 2003;138:215-229.

36. Tight blood pressure control and risk of macrovascular and microvascular complications in type 2 diabetes: UKPDS 38. UK Prospective Diabetes Study Group. BMJ. 1998;317:703-713. 